\title{
A Analytical Portend To Increase Energetic Packet Breakdown Determination
}

\author{
Ph.D. Sangeeta Bhatia \\ Muthoot Institute Of Technology \& Science India
}

\begin{abstract}
Determination and reasoning of packet breakdown options area unit exigent thanks to the moderately uncommon incidence and generally short period of packet breakdown episodes. The intention of our study is to grasp a way to verify packet breakdown episodes accurately with end to-end probes. we have a tendency to begin by burdensome the power of normal Poissonmodulated finish-to-end determinations of breakdown during a prohibited laboratory surroundings mistreatment scientific discipline routers and repair end hosts. Our tests show that breakdown characteristics reported from such Poisson modulated probe tools will be pretty incorrect over a series of traffic conditions. Aggravated by these annotations Specifically, our methodology entails probe tests that follow a applied math allocation to 1) allow a definite tradeoff between accuracy and force on the network, and 2) allow a lot of correct determinations than traditional Poisson searching at a similar rate. we have a tendency to appraise the capabilities of our slant textually by mounting and implementing a model tool, known as V OILA. The tests categorical the trade-offs between force on the network and determination accuracy. we have a tendency to show that V OILA hearsay breakdown characteristics much more accurately than typical breakdown determination tools.
\end{abstract}

Keywords: Energetic Determination, VOILA, network congestion, network probes, packet failure.

\section{Introduction}

Determinative and analyzing network traffic dynamics between finish hosts has provided the institution for the growth of many special network protocols and systems. Of fussy consequence understands packet breakdown behavior since breakdown will have a big impact on the concert of each TCP- and UDP-based applications. despite labor of network engineers and operators to certain failure, it'll nearly actually ne'er be eliminated thanks to the essential dynamics and scaling properties of transfer in packet switched network [1]. Network operators have the gift to reflexively observe nodes inside their network for packet breakdown on routers mistreatment 
SNMP. End-to-end energetic determinations mistreatment probes offer a uniformly precious outlook since they signify the circumstances that application transfer is experiencing on those methods. the foremost often used tools for searching finish to end methods to work out packet breakdown fit the ubiquitous PING operate.

We begin by testing customary Poisson modulated searching during a prohibited and suspiciously instrumented laboratory surroundings consisting of artefact workstations separated by a series of scientific discipline routers. Background traffic is shipped between finish hosts at completely different levels of intensity to get breakdown episodes thereby sanctionative repeatable tests over a variety of conditions. we have a tendency to contemplate this setting to be ideal for testing breakdown determination tools since it combines the benefits of ancient simulation environments with those of tests within the wide space. Namely, very like simulation, it provides for a high level of management and a capability to match results with "ground truth." what is more, very like tests within the wide space, it provides a capability to contemplate breakdown processes in actual router buffers and queues, and also the behavior of implementations of the tools on artefact finish hosts. Our tests expose 2 imperative deficiencies with straightforward Poisson searching.

\section{Related work}

There are several studies of packet breakdown behavior within the net. Bolot [6] and Paxson [7] evaluated end-to-end probe determinations and reported characteristics of packet breakdown over a range of methods within the wide space. Yajnik et al. evaluated packet breakdown correlations on longer time scales and developed Andre Markoff models for written record addiction structures [8]. Zhang et al. characterized many aspects of packet breakdown behavior [5]. specially, that employment reported determines of responsibility of breakdown episode rate, breakdown episode period, breakdown free amount period and overall breakdown rates. Papagiannaki et al. [9] used a complicated passive observance infrastructure within Sprint's scientific discipline backbone to congregate packet traces and analyze characteristics of delay and congestion. Finally, the restrictions in customary end-to-end Poisson searching tools by comparison the breakdown rates determined by such tools to breakdown rates determined by passive means that during a absolutely instrumented wide space infrastructure

\section{Definitions of breakdown characteristics}

Ph.D. Sangeeta Bhatia 
There area unit several factors which will contribute to packet breakdown within the net. we have a tendency to illustrate a number of these problems in specify as a basis for accretive our energetic determination objectives. The background that we have a tendency to contemplate is sculpturesque as a collection of flows that exceed through a router $\mathrm{R}$ and contend for one output link with information measure Bout as shown in Fig. 1(a). The additive input information measure (Bin) should be larger than the collective output link (Bout) so as for breakdown to require place. The mean trip time for the $\mathrm{N}$ flows is $\mathrm{M}$ sec. Router $\mathrm{R}$ is organized with $\mathrm{Q}$ bytes of packet buffers to place up transfer bursts, with $Q$ naturally sized on the order of M B [20], [21]. we have a tendency to presume that the queue operates during a first in first out methodology, that the traffic includes a concoction of short- and long-lived transmission control protocol flows as is common in today's net, which the worth of can ebb and flow over time.

\section{Estimation of plain poisson searching for packet breakdown}

We begin by mistreatment our laboratory to estimate the capabilities of straightforward Poissonmodulated breakdown probe determination mistreatment the ZING tool [17], [18]. ZING determines packet waiting associate degreed breakdown in one direction on an end-to-end path. The ZING sender sends UDP probe packets at Poisson modulated mean times with timestamps and distinctive serial numbers and also the receiver logs the probe packets incoming. Users determine the mean probe rate, the probe packet size, and also the range of packets during a "fledge." To estimate straightforward Poisson searching, we have a tendency to organized ZING mistreatment a similar parameters as in [5]. Specifically, we have a tendency to trotted 2 tests, one with $=200 \mathrm{~ms}(10 \mathrm{~Hz})$ and 512 computer memory unit payloads and another with $=100 \mathrm{~ms}$ ( 20 $\mathrm{Hz}$ ) and 128 computer memory unit payloads. to work out the extent of our tests below, we have a tendency to most well-liked associate degree epoch of your time that ought to certain the distinction of the breakdown rate calculator $X$ wherever $\operatorname{Var}(n X) \approx p / n$ for breakdown rate $p$ and range of probes $n$.

\section{Probe tool performance and estimation}


To estimate the capabilities of our breakdown probe determination method, we have a tendency to designed a tool known as VOILA1 that tack the essential algorithmic rule. we have a tendency to then conducted a sequence of experiments with VOILA in our laboratory with a similar background traffic situations delineate in Section IV.We investigated the primary hypothesis during a sequence of tests mistreatment the infinite transmission control protocol supply background traffic and constant-bit rate traffic delineate in Section IV. supposed of the infinite transmission control protocol traffic, breakdown event durations were roughly $150 \mathrm{~ms}$. For the constant-bit rate traffic, breakdown episodes were roughly sixty eight ms in period. we have a tendency to used a bespoken edition of VOILA to supply probes at permanent intervals of ten ms in order that some range of probes would come upon all breakdown episodes. we have a tendency to tested with probes consisting of between one and ten packets. Packets in a personal probe were sent back to back per the capabilities of the determinant hosts (i.e., with roughly thirty $\mu$ s between packets). Probe packet sizes were set at 600 computer memory unit.

\section{Using voila in follow}

There area unit variety of necessary realistic problems that should be thought-about once mistreatment VOILA within the wide area: The tool needs the user to pick out values for $p$ and $\mathrm{N}$. Assume for currently that the quantity of breakdown events is immobile over time. Let B zero be the mean range of breakdown events that occur over a unit amount of your time. as an example, if an \{everyday\} of twelve breakdown events occur every minute, and our discretization unit is five $\mathrm{ms}$, then $\mathrm{B} 0$

$=12 /(60 \times 200)=z e r o .001$ (this is, of course, associate degree estimate of actuality the worth of $B$ zero ). With the inactive assumption on B zero, we have a tendency to imagine the accuracy of our evaluators to rely on the merchandise $p N B$ zero, however not on the individual values of $p$ , $\mathrm{N}$ or $\mathrm{B} 0$. Indeed, that a dependable rough calculation of the relative variance in our estimation of period is given by Standard Deviation (duration)

$1 / 2 \mathrm{pNB}$

The recent study on packet breakdown via passive determinant [9] indicates that breakdown episodes in backbone links will be terribly transient (e.g., on the order of many microseconds).

Summary, conclusions and future work

Ph.D. Sangeeta Bhatia 


\section{THE AMERICAN JOURNAL OF}

ENGINEERING AND TECHNOLOGY

The intention of our study was to acknowledge verify end-to-end packet breakdown individuality accurately with probes and during a way that allows USA to specify the impact on the bottleneck queue. we have a tendency to began by estimating the skills of straightforward Poissonmodulated searching during a controlled laboratory surroundings consisting of artefact finish hosts and scientific discipline routers. we predict concerning this for breakdown determination tool analysis since it activates repeatability, concern of ground truth, and a variety of traffic conditions below that to subject the tool. Our primary tests illustrate that easy Poisson searching is comparatively ineffective at activity breakdown episode frequency or determinative breakdown episode period, significantly once subjected to transmission control protocol (immediate) cross traffic.

\section{Acknowledgement}

We, authors categorical feeling to all or any the anonymous reviewers for his or her affirmative annotations among our paper.

\section{References}

[1] W. Leland, M. Toque, W. Dillinger, and D. Wilson, "On the self- similar nature of local area network traffic (extended version)," IEEE/ACM Trans.Networking.

[2] J. Bolot, "End-to-end packet delay and loss behavior within the net," in Proc. ACM SIGCOMM '93, urban center, CA, Sep.

[3] D. Papagiannaki, R. Cruz, and C. Diet, "Network performance observance at little time scales," in Proc. ACM SIGCOMM '03, Miami, FL, Oct.

[4]S. Brummell, "On the connection between client and time averages in queues," J. Appl. Probable.

[5]S. Aloof, P. Nain, and D. Owsley, "Inferring network characteristics via moment-based estimators," in Proc. IEEE INFOCOM '01, Anchorage, AK, Apr. 


\section{THE AMERICAN JOURNAL OF}

ENGINEERING AND TECHNOLOGY

\section{VOLUME01 ISSUE02}

[6]K. Dalmatian, B. Banta, and T. Bugnazet, "Cross traffic estimation by loss method analysis," in Proc. ITC Specialist Seminar on net Traffic Engineering and Traffic Management, Wurzburg, Germany, Jul. 2007. 